6

\title{
Automatic checking of the compatibility of the ISDN user-network interface (layer 3) using the tool VACCIN
}

\author{
Pierre-Yves DANET \\ France Telecom CNET \\ Dept. LAA/TCS/TRA \\ Technopôle Anticipa, 2 avenue Pierre Marzin, F-22307 LANNION \\ Cedex FRANCE \\ Tel : +339605 I1 55, Fax : +3396050242 \\ E-Mail : pierre-yves.danet@lannion.cnet.fr
}

\begin{abstract}
This paper presents the results of a study which aim was to develop a tool able to detect automatically incompatibilities between ISDN terminals already used and a futur version of the network.
\end{abstract}

ISDN, SDL, user-network interface

\section{Keywords}

\section{INTRODUCTION}

The introduction of new versions of software for the ISDN switching system presents a compatibility problem with the terminals already in operation. The aim of the telecom operator is the non-disruption of the operating terminals during the introduction of new services on the network. The testing of terminals (even with certification) is not sufficient to provide a total guarantee.

Instead of considering very expensive corrective measures - the development of terminal software or the introduction of network corrections - this study attempts to provide preventive measures using simulation techniques.

Previous experience has shown the limits of a "manual" analysis between two versions of a specification. CNET has therefore examined the possibility of automatically verifying this compatibility by using a tool capable of "exhaustively" simulating all the terminal-network exchanges (layer 3 of ISDN : CSE P 22-30A [1] for the terminal side \& ETS 300 403-1 [3] for the network side). But the high number of simulations required make it impossible to carry out an exhaustive study. 
This new approach consists in verifying the compatibility of the network-terminal interface specifications by using formal specifications (SDL 1992 [5] - Specification and Description Language). The first objective of this study involves the development of a simulator on the basis of the GEODE tool (v.2) from Verilog. This will make it possible to achieve simulations with reference to the formal descriptions included in Terminal and Network specifications.

The various problems which are likely to arise are:

- message not known,

- information element not known,

- field value of information element not known,

- different interpretation of a value,

- automaton modification.

On this basis the tool should be capable of achieving the simulation of the establishment and release of a call by systematically scanning all the coding combinations allowed by the standard (Ref.4.2, page 6). The possible combinations are so numerous that we have introduced an option which allows only the scanning of those coding combinations corresponding to the existing terminal types (telephone, adapter, fax, videophone, ...).

\section{TECHNICAL DESCRIPTION}

\subsection{Development of a simulator}

This model is based on the GEODE simulator and uses in particular the SDL descriptions for the network and the terminal specifications, as follows :

- SDL specification of version Vn of the network side protocol,

- SDL specification of version Vn-1 of the terminal side protocol,

- Description of the message coding and of the information elements of version $\mathrm{Vn}$,

- Description of the message coding and of the information elements of version $\mathrm{Vn}-1$. quality.

To obtain significant simulation results the initial data listed above must be of the highest

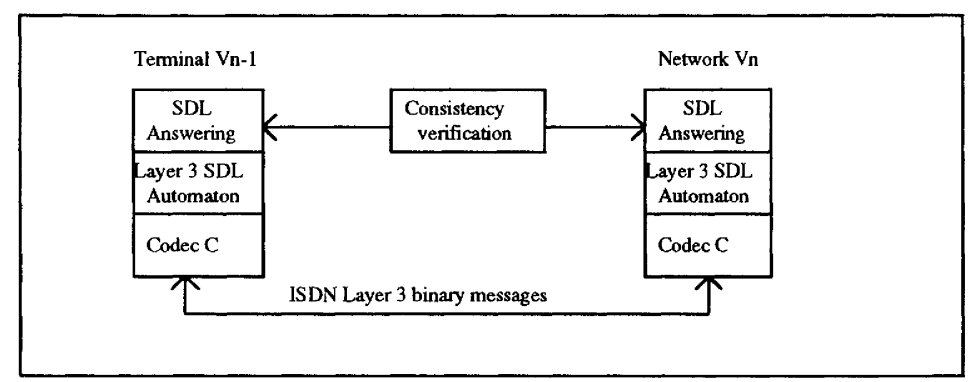

Figure 1 Functional description of the system.

Role of each object:

The SDL answering systems activate the SDL automata using predefined scenarios. Each scenario consists of a series of layer 3 service primitives. These primitives indicate the ISDN 
messages to be transmitted or received by the automaton. It is possible to fix or vary the value of the information elements for each service primitive. The answering systems, the service primitives and the information elements are implemented in SDL. At present the scenarios involve call establishment and release. At a later stage they could be designed to simulate the implementation of supplementary services.

The layer 3 SDL automata carry out the ISDN communication according to protocol D of standard $\mathrm{Vn}-1$ on the terminal side or the standard $\mathrm{Vn}$ for the network. The automata deal with the ISDN messages and the information elements using SDL internally, but exchange them in binary form. In order to do that the automata use codec C.

The codecs $C$ change the SDL information elements and the ISDN messages transmitted by the automata into binary code and decode the binary messages received by the automata into SDL information elements and ISDN messages. The codecs $\mathrm{C}$ have been developed in $\mathrm{C}$ language.

The operator starts the simulation by activating the SDL answering systems. The aim of the simulation is to detect interworking problems between the terminal Vn-1 and the network $\mathrm{Vn}$ in relation to exchanges of ISDN messages and ncoding / decoding of messages and information elements.

The answering systems also have to verify the reliability of the scenario (the establishment or release of a call), as well as verifying the compatibility of the value interpretation. In fact, a value could have different interpretations depending on the protocol version in use.

\subsection{Development of an answering system 2.2.1. Aim of the answering system}

The answering systems (one terminal side and one network side) must allow the activation of the overall SDL system and the automatic operation of the simulation. They allow the modelling of the behaviour of the application oriented layer of an actual terminal (to make a call, to answer a call, to release a call). The answering systems are designed under the form of SDL automata.

The aim of the answering systems is to generate simulation scenarios which must link the establishment and release of the calls, using, if necessary, all the parameter values associated with the layer 3 primitives, i.e. all the fields of information elements which make up the message exchanges between the layer 3 automata.

The answering system is composed of two distinct parts:

- One part consists of a module of predefined scenarios, i.e. a module of sequences of the layer 3 service primitives; the progress of a scenario chosen by the operator supposes the execution of a scenario $\mathrm{Vn}-1$ on the terminal side and the execution of a scenario $\mathrm{Vn}$ on the network side (a sub-set of predefined scenarios exists on both sides).

- The other part is for the activation and parameterization of these scenarios:

- the activation involves selecting a scenario and linking different scenarios if necessary; it also involves synchronising the two scenarios which are carried out on both the terminal side and the network side.

- the parameterization is carried out either by fixing the parameter values of the layer 3 service primitives, or by scanning these values, i.e. by repeating the same scenario many times with different parameters; the simulation can be done completely automatic. 


\subsubsection{Presentation of the functional modules of the answering system}

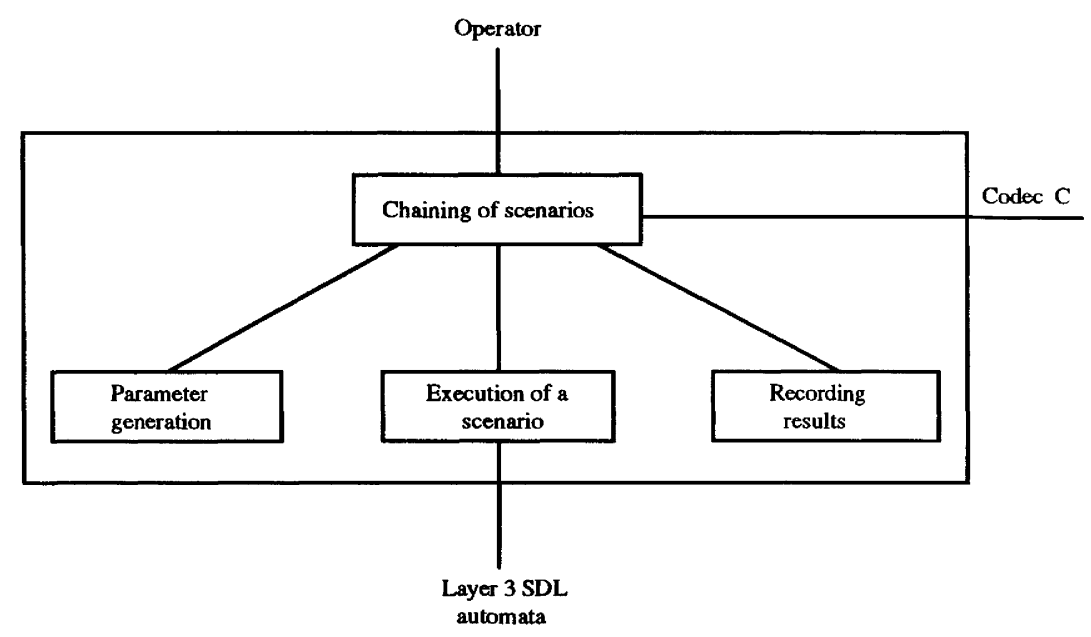

Figure 2 Link description between each function.

The answering system offers the following functions :

- Linking of scenarios : activating and synchronising the scenarios, with a scanning of parameter values if necessary, and the initialisation/termination of the codecs.

- Execution of a scenario : activation of the layer 3 automaton concerned, in accordance with the linking of the anticipated service primitives in the scenario and their parameterization. This could be scenario Vn-1 on the terminal side or scenario $\mathrm{Vn}$ on the network side.

- Generation of parameters : generation of layer 3 service primitives in order to give all possible combinations of information elements for each layer 3 message and for all the possible values of each of the fields of information elements. This could be service primitives $\mathrm{Vn}-1$ on the terminal side or $\mathrm{Vn}$ on the network side.

- Recording of results : recording of the scenarios and the parameterization which were carried out, as well as the results (OK or NOK) in a trace file. 


\subsection{Development of a codec system}

\subsubsection{Aim of the codec system C}

The aim of the decoder is to decode the binary messages according to a given specification and to detect non-conformity in relation to this specification.

The aim of the encoder is to encode the SDL message according to a given specification.

\subsubsection{Presentation of the functional modules of the codec system}

The diagram below shows the functions of the codec system.

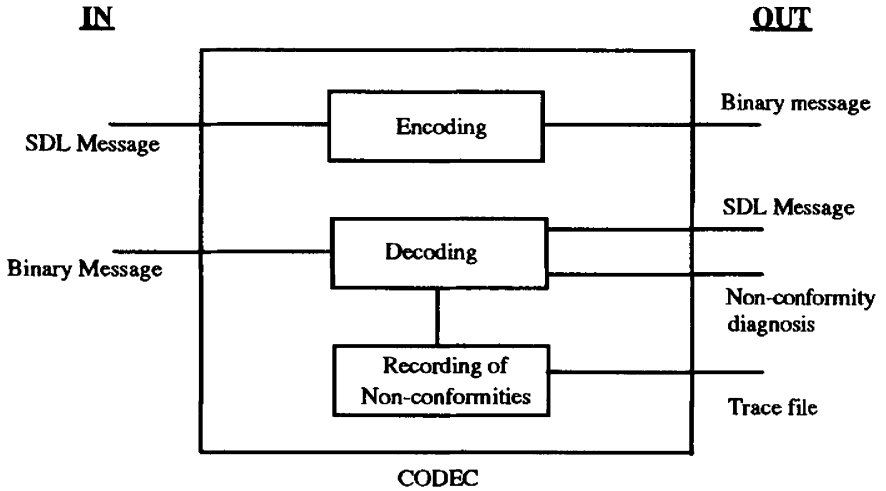

Figure 3 Description of the input/output information.

The codec system offers the following functions :

- Encoding process Vn-1

- Decoding process $\mathrm{Vn}-1$

- Recording of non-conformities Vn-1

- Encoding process $\mathrm{Vn}$

- Decoding process Vn

- Recording of non-conformities Vn

\subsection{Implementation of the simulations}

The simulation is started when the operator activates the SDL answering systems. Its aim is to detect the non-conformities between the terminal $\mathrm{Vn}-1$ and the network $\mathrm{Vn}$ in relation to exchanges of ISDN messages (using the answering systems and layer 3 automata) and encoding / decoding of messages and information elements (using the answering systems, layer 3 automata and codecs).

The simulator actually uses $\mathrm{C}$ language to encode the information elements. In future it would be more judicious to use ASN.1, but this will require a considerable amount of work. 
The ultimate stage will involve the analysis of the incompatibilities. This will make it possible to detect those which will be likely to prevent the interworking of the new version of the network with a given terminal.

\section{CONCLUSION}

The results of the simulation were very useful because the analysis of them shows us some new problems which were not identified "manually". Hopefully these "new" incompatibilities were not so serious because error management procedures described in the ISDN layer 3 protocol [1] are able to handle them.

This first experiment was very useful because it gave us the following information :

-Manual" checking of protocol compatibility cannot be exhaustive.

- Automatic checking is much faster (1 month instead of 5 months).

- Automatic checking has to be improved in order to include help for results analysis.

- Automatic checking has to be improved in order to use terminal profiles representing exactly the behaviour of one terminal instead of using a general profile (complete specification).

Then we need to work into 3 directions :

- improvement of the result function in order to help the operator to identify the incompatibilities,

- use of ASN.1 [4] for the description of messages and information elements,

- development of a tool able to generate a specific terminal profile from the layer 3 specification [3] and from the PICS (Protocol Implementation Conformance Statement) and the PIXIT (Protocol Implementation eXtra Information for Testing) values.

\section{DEFINITIONS - REFERENCES}

\subsection{Glossary}

ISDN : Integrated Services Digital Network

Vn : Version $n$

\subsection{References}

[1] ETS 300 403-1 : ISDN; DSS1; Signalling network layer for circuit-mode basic call control; part 1 : protocol specification

[2] ETS 300 403-2 : ISDN; DSS 1; Signalling network layer for circuit-mode basic call control; part $1:$ SDL

[3] CSE P 22-30 : Layer 3 of call control protocol D for access to ISDN

[4] ASN.1 : Abstact Syntax Notation One (IS 8824 \& IS 8825 1988)

[5] SDL : Specification and Description Language (Z.100 1992) 